\title{
Thermochromatography of Platinum Elements in Oxygen: Radiochemical Studies of the Behaviour of Rhodium, Palladium, Osmium and Platinum
}

\author{
By F. Zude', W. Fan ${ }^{1,2 *}$, N. Trautmann', G. Herrmann ${ }^{1,2}$ and B. Eichler ${ }^{3}$ \\ ' Institut für Kernchemie, Universität Mainz, D-55099 Mainz, Germany \\ 2 Gesellschaft für Schwerionenforschung, D-64220 Darmstadt, Germany \\ ${ }^{3}$ Paul Scherrer Institut, CH-5232 Villigen, Switzerland
}

(Received October 6, 1992)

Thermochromatography / Platinum elements, thermochromatographic separation / Rhodium oxides, volatile / Platinum oxides, volatile

\begin{abstract}
The volatilization of rhodium, palladium, osmium and platinum in air and oxygen and their subsequent deposition in a temperature gradient tube was investigated with carrier-free radioisotopes and labelled macroamounts. Palladium remains at the starting position, rhodium forms a deposit at high and platinum one at medium temperature, and osmium passes through the column.
\end{abstract}

\section{Introduction}

Thermochromatography is a very useful radiochemical separation technique [1]: Volatile species are separated in a column with a negative temperature gradient by deposition in zones of different temperatures. For carrier-free species the adsorption enthalpy on the stationary phase governs the deposition. Thermochromatographic separations can be performed on a time scale of a few seconds and, hence, play an important role in investigations of short-lived nuclides and very heavy elements. This was our motivation to continue previous studies $[2,3]$ on the behaviour of platinum elements in a temperature gradient tube using dry and moist air and oxygen as the reactive carrier gases. Platinum elements are supposed to be homologs of the transactinide elements 108 to 110 [4] and, thus, may serve to explore methods for studying the chemistry of such elements.

We have already reported our results for ruthenium [5] and iridium [6] where transport reactions with a change of the oxidation state:

$$
\mathrm{MeO}_{3}(\mathrm{~g}) \leftrightarrow \mathrm{MeO}_{2} \text { (s or ads) }+1 / 2 \mathrm{O}_{2}
$$

were identified as major processes, with contributions of other species, $\mathrm{RuO}_{4}$ and $\mathrm{H}_{2} \mathrm{IrO}_{4}$, under certain

\footnotetext{
* Present address: Nuclear Medicine Division, The Second Affiliation Hospital, Suzhou Medical College, PRC-215004 Suzhou.
}

conditions. The behaviour of ruthenium and iridium were treated in detail $[5,6]$ in order to show how reaction mechanism and relevant thermodynamic data can be deduced in such more complicated cases. In the following note we report results for the other four platinum elements - rhodium, palladium, osmium, and platinum - studied in form of carrier-free radioisotopes and labelled macroscopic amounts.

\section{Experimental}

Our thermochromatographic setup is described elsewhere [5] in detail. A decreasing temperature gradient was produced along the chromatographic column by winding the heating wire in logarithmically increasing distances. The cold end was cooled with methanol-dry ice or with liquid nitrogen. Downstream of the cooled part the exhaust gases passed a charcoal trap. The chromatographic columns were quartz tubes $105 \mathrm{~cm}$ long, $3 \mathrm{~mm}$ inner diameter ( $\mathrm{Rh}, \mathrm{Pd}$, Os experiments) or 50 to $60 \mathrm{~cm}$ long, $5 \mathrm{~mm}$ i.d. $(\mathrm{Rh}, \mathrm{Pt})$. They were used empty or filled with quartz powder of selected

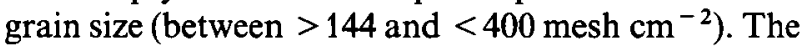
samples were placed in the hottest part of the column in form of deposits on quartz wool. Starting temperatures were 1320 or $1400 \mathrm{~K}$ with a temperature gradient of $8 \mathrm{~K} \mathrm{~cm}^{-1}$ in the long, and 1270 to $1460 \mathrm{~K}$ with 17 or $33 \mathrm{~K} \mathrm{~cm}^{-1}$ gradients in the short columns. Air and oxygen were used as carrier gases at flow rates of 42 to $72 \mathrm{l} \mathrm{h}^{-1}$ in the long and 10 to $20 \mathrm{l} \mathrm{h}^{-1}$ in the short columns. The carrier gases were loaded with water vapour at known partial pressure up to $610 \mathrm{~Pa}$. Exposure times varied between 5 and $120 \mathrm{~min}$.

For the production of labelled macroscopic samples $\mathrm{Pd}\left(\mathrm{NO}_{3}\right)_{2}, \mathrm{~K}_{2} \mathrm{OsO}_{4}, \mathrm{PtCl}_{4}$ or $\mathrm{Pt}\left(\mathrm{NO}_{3}\right)_{4}$ were irradiated with thermal neutrons in the Mainz research reactor. The radiotracers were $13.5-\mathrm{h}{ }^{109} \mathrm{Pd}, 30.5-\mathrm{h}$ and $15.4-\mathrm{d}^{191} \mathrm{Os}, 18.3-\mathrm{h}{ }^{197} \mathrm{Pt}$ and $4.0-\mathrm{d}{ }^{195 \mathrm{~m}} \mathrm{Pt}$. Solutions of the irradiated compounds were dried on $20 \mathrm{mg}$ of quartz wool and introduced into the column without further treatment except for $\mathrm{K}_{2} \mathrm{OsO}_{4}$ which was reduced to the metal with hydrogen before the thermochromatographic experiment was started. 
Carrier-free 35.4 -h ${ }^{105} \mathrm{Rh}$ was obtained by $\beta^{-}$-decay of 4.44-h ${ }^{105} \mathrm{Ru}$ after neutron activation of $\mathrm{Ru}\left(\mathrm{NO}_{3}\right)_{4}$ and was applied without separation from the bulk of ruthenium, typically 0.1 to $0.3 \mathrm{mg}$ of the irradiated compound. Alternatively carrier-free 21.7$\min { }^{107} \mathrm{Rh}$ from neutron-induced fission of ${ }^{249} \mathrm{Cf}$ was used after an on-line collection of fission products as described elsewhere [5] and $\beta^{-}$-decay of its parent 3.8$\min { }^{107} \mathrm{Ru}$ during a waiting period. Carrier-free $3.0-\mathrm{d}$ ${ }^{191} \mathrm{Pt}$ was produced by the ${ }^{190} \mathrm{Os}(\alpha, 3 \mathrm{n})$ reaction at the cyclotron of the Kernforschungszentrum Karlsruhe by bombarding pellets of $400 \mathrm{mg}$ osmium powder, $8 \mathrm{~mm}$ in diameter, with $37 \mathrm{MeV} \alpha$-particles. After dissolution in hot concentrated nitric acid $\mathrm{OsO}_{4}$ was distilled off in presence of hydrogen peroxide. Aliquots of the resulting stock solution of ${ }^{191} \mathrm{Pt}$ were mixed with $\operatorname{Pt}\left(\mathrm{NO}_{3}\right)_{4}$ carrier to obtain samples with known low platinum content. Preconditioning as used for iridium [6] was also applied in some experiments. In these cases the platinum sample was heated to $1420 \mathrm{~K}$ in oxygen and the volatilized fraction collected in the quartz column in a steep temperature gradient. The deposit was then reduced in dry hydrogen at $930 \mathrm{~K}$ and used in the chromatographic experiment.

After exposure the activity distribution along the thermochromatographic column was measured in $2 \mathrm{~cm}$ sections by $\gamma$-ray spectroscopy with $\mathrm{Ge}(\mathrm{Li})$ detectors placed in a lead collimator. The charcoal trap was also measured.

\section{Results and discussion}

\subsection{Rhodium}

Efficient volatilization, with over $90 \%$ yield, of carrierfree rhodium in dry oxygen from irradiated $\mathrm{Ru}\left(\mathrm{NO}_{3}\right)_{4}$ requires high starting temperatures, $1460 \mathrm{~K}$, and long exposure times, two hours. Rhodium is deposited around $1060 \pm 50 \mathrm{~K}$ in an empty and $1120 \pm 70 \mathrm{~K}$ in a filled column. In dry air at $1400 \mathrm{~K}$ starting temperature the volatilization proceeds even more slowly with some improvement by using moist air. The deposition zone remains at $1030 \pm 70 \mathrm{~K}$. With carrier-free rhodium from fission the results are essentially the same. At a starting temperature of $1330 \mathrm{~K}$ about $50 \%$ are volatilized within 10 to $15 \mathrm{~min}$ in dry and moist oxygen and are deposited around $1060 \mathrm{~K}$. No volatilization is observed at $1210 \mathrm{~K}$ starting temperature. The deposition zones are always of normal width, in contrast to previous observations [3] in on-line experiments where broad zones extending down to about $300 \mathrm{~K}$ appeared.

\subsection{Palladium}

No volatilization of macroscopic amounts of palladium applied as the nitrate was observed in dry or moist air at starting temperatures up to $1400 \mathrm{~K}$. Online experiments with carrier-free palladium [3] did also not yield evidence for volatilization.

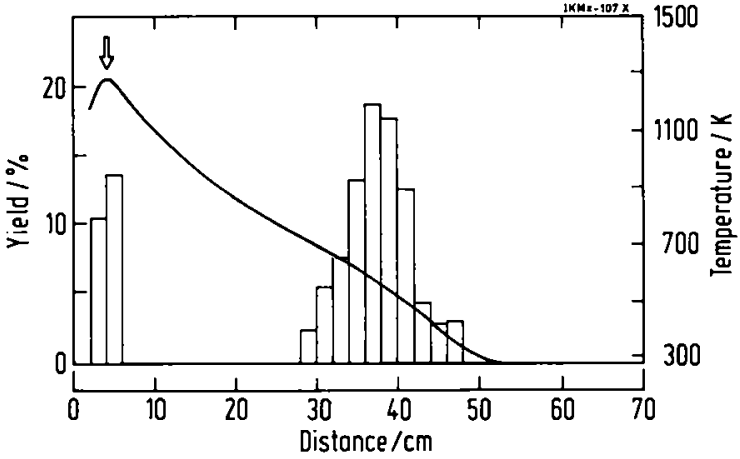

Fig. 1. Thermochromatogram of carrier-free platinum in moist oxygen: Pretreated sample, empty column, partial pressure of water vapour $74 \mathrm{~Pa}$, flow rate $101 \mathrm{~h}^{-1}$, exposure time $30 \mathrm{~min}$. The solid line gives the temperature along the column, righthand scale, the arrow indicates the starting position.

\subsection{Osmium}

Osmium is nearly completely volatilized in dry and moist air, as expected for its very volatile and stable tetroxide. Most of the osmium is transported to the charcoal trap with some deposition in the cooled part of the column. This is in agreement with previous observations with carrier-free samples [3] and with the low adsorption enthalpy, 40 to $57 \mathrm{~kJ} \mathrm{~mol}^{-1}[3,7]$, of $\mathrm{OsO}_{4}$ on quartz deduced from thermochromatographic experiments.

\subsection{Platinum}

Macroscopic amounts of platinum, beyond $0.4 \mathrm{mg}$, in the form of $\mathrm{PtCl}_{4}$ and $\mathrm{Pt}\left(\mathrm{NO}_{3}\right)_{4}$ are not volatile in dry nor in moist air. This has also been observed for metallic platinum treated with dry air [2]. With carrierfree samples a single, broad deposition zone at about $500 \mathrm{~K}$ is found in dry oxygen. After preconditioning of the platinum sample the zone becomes narrower and is located at $535 \pm 55 \mathrm{~K}$ in dry and $555 \pm 50 \mathrm{~K}$ in moist oxygen. Figure 1 shows a chromatogram in moist oxygen. Only a single zone is observed. This is in contradiction with Refs. $[2,3]$ where a second zone around $330 \mathrm{~K}$ appeared as the minor zone in offline [2] and the major zone in on-line experiments [3]. On the whole, the behaviour of platinum is quite sensitive to experimental details as is also noted in Ref. [3]. In the transition region between microscopic and macroscopic amounts, the deposition temperature after $30 \mathrm{~min}$ exposure in dry oxygen remains at $530 \pm 80 \mathrm{~K}$ for very low concentrations until a tracer amount of $1 \mu \mathrm{g}$ is reached. Then it moves gradually to $700 \pm 50 \mathrm{~K}$ at $0.1 \mathrm{mg}$ tracer. This is very similar to the behaviour of iridium [6]. It may have the same origin, the transition from adsorption of microscopic to desublimation of macroscopic amounts at about $1 \mu \mathrm{g}$ of carrier when a pure solid phase can be formed. In contrast to iridium the volatilization yield of platinum decreases steadily with increasing amount and ceases 
beyond about $0.1 \mathrm{mg}$. The location of the platinum deposition zone in our study agrees well with the hightemperature zone observed in air [2,3] and may be explained, in analogy to iridium [6], by a transport reaction of gaseous $\mathrm{PtO}_{3}$ with deposition as $\mathrm{PtO}_{2}$ according to reaction (1).

\section{Acknowledgements}

We are grateful for numerous irradiations to $\mathrm{K}$. $\mathrm{H}$. Assmus and the staff of the Karlsruhe cyclotron and to the staff of the Mainz research reactor.

\section{References}

1. Zvara, I.: Isotopenpraxis 26, 251 (1990).

2. Domanov, V. P., Eichler, B., Zvara, I.: Radiokhimija 26, 66 (1984); Engl. transl. Sov. Radiochem. 26, 63 (1984).

3. Domanov, V. P., Zvara, I.: Radiokhimija 26, 770 (1984); Engl. transl. Sov. Radiochem. 26, 731 (1984).

4. Herrmann, G.: Angew. Chem. 100, 1471 (1988); Engl. transl. Angew. Chem. Int. Ed. 27, 1417 (1988)

5. Eichler, B., Zude, F., Fan, W., Trautmann, N., Herrmann, G.: Radiochim. Acta 56, 133 (1992).

6. Eichler, B., Zude, F., Fan, W., Trautmann, N., Herrmann, G.: Radiochim. Acta 61, 81 (1993).

7. Eichler, B., Domanov, V. P.: J. Radioanal. Chem. 28, 143 (1975). 
